Bull. Korean Math. Soc. 48 (2011), No. 1, pp. 157-167

DOI 10.4134/BKMS.2011.48.1.157

\title{
RIGIDNESS AND EXTENDED ARMENDARIZ PROPERTY
}

\author{
Muhittin Başer, Fatma Kaynarca, and Tai Keun Kwak
}

\begin{abstract}
For a ring endomorphism $\alpha$ of a ring $R$, Krempa called $\alpha$ a rigid endomorphism if $a \alpha(a)=0$ implies $a=0$ for $a \in R$, and Hong et al. called $R$ an $\alpha$-rigid ring if there exists a rigid endomorphism $\alpha$. Due to Rege and Chhawchharia, a ring $R$ is called Armendariz if whenever the product of any two polynomials in $R[x]$ over $R$ is zero, then so is the product of any pair of coefficients from the two polynomials. The Armendariz property of polynomials was extended to one of skew polynomials (i.e., $\alpha$-Armendariz rings and $\alpha$-skew Armendariz rings) by Hong et al. In this paper, we study the relationship between $\alpha$-rigid rings and extended Armendariz rings, and so we get various conditions on the rings which are equivalent to the condition of being an $\alpha$-rigid ring. Several known results relating to extended Armendariz rings can be obtained as corollaries of our results.
\end{abstract}

Throughout this paper, all rings are associative with identity. Given a ring $R$, the polynomial ring over $R$ is denoted by $R[x]$. Recall that a ring $R$ is called reduced if it has no nonzero nilpotent elements. Armendariz $[1$, Lemma 1] showed that for a reduced ring $R$, if any polynomial $f(x)=a_{0}+a_{1} x+\cdots+$ $a_{m} x^{m}, g(x)=b_{0}+b_{1} x+\cdots+b_{n} x^{n} \in R[x]$ satisfies $f(x) g(x)=0$, then $a_{i} b_{j}=0$ for each $i, j$. Since then, Rege and Chhawchharia [12] called $R$ an Armendariz ring if it satisfies this condition. Many properties of Armendariz rings have been studied by many authors $[2,3,5,6,8,10,11,12]$.

The reducedness and Armendariz property of a ring were extended as follows. For a ring $R$ with a ring endomorphism $\alpha: R \rightarrow R$, a skew polynomial ring (also called an Ore extension of endomorphism type) $R[x ; \alpha]$ of $R$ is the ring obtained by giving the polynomial ring over $R$ with the new multiplication $x r=\alpha(r) x$ for all $r \in R$. Recall that an endomorphism $\alpha$ of a ring $R$ is called rigid [9] if $a \alpha(a)=0$ implies $a=0$ for $a \in R$, and a ring $R$ is called $\alpha$-rigid [4] if there exists a rigid endomorphism $\alpha$ of $R$. Note that any rigid endomorphism of a ring is a monomorphism, and $\alpha$-rigid rings are reduced rings [4, Proposition 5]. On the other hand, the Armendariz property with respect to polynomials was extended to one of skew polynomials. A ring $R$ is called $\alpha$-Armendariz (resp.,

Received June 2, 2009.

2010 Mathematics Subject Classification. 16S36, 16U50,16W20.

Key words and phrases. reduced rings, skew polynomial rings, rigid rings, (extended) Armendariz rings, trivial extension, semiprime rings, semicommutative rings. 
$\alpha$-skew Armendariz) [6, Definition 1.1] (resp., [5, Definition]) if for $p(x)=$ $a_{0}+a_{1} x+\cdots+a_{m} x^{m}$ and $q(x)=b_{0}+b_{1} x+\cdots+b_{n} x^{n}$ in $R[x ; \alpha], p(x) q(x)=0$ implies $a_{i} b_{j}=0$ (resp., $a_{i} \alpha^{i}\left(b_{j}\right)=0$ ) for all $0 \leq i \leq m$ and $0 \leq j \leq n$. It can be easily checked that every subring $S$ with $\alpha(S) \subseteq S$ of an $\alpha$-Armendariz ring (resp., an $\alpha$-skew Armendariz ring) is also $\alpha$-Armendariz (resp., $\alpha$-skew Armendariz). Note that every $\alpha$-rigid ring is $\alpha$-Armendariz [6, Proposition 1.7], and every $\alpha$-Armendariz ring is $\alpha$-skew Armendariz [6, Theorem 1.8], but the converses do not hold by [6, Example 1.6] and [6, Example 1.9], respectively. Moreover $R$ is an $\alpha$-rigid ring if and only if $R[x ; \alpha]$ is reduced [5, Proposition 3]. In [3], Chen and Tong showed the relationship between $\alpha$-rigid rings and $\alpha$ skew Armendariz rings. Motivated by their results, in this paper, we continue the study of $\alpha$-Armendariz rings, improving several results in [3] and [6], and moreover we obtain various rings which are equivalent to $\alpha$-rigid rings. Several known results relating to Armendariz rings can be obtained as corollaries of our results.

In [12, Remark 3.1], Rege and Chhawchharia showed that every $n \times n$ full matrix ring over any $\operatorname{ring} R$ is not $I_{R}$-Armendariz for $n \geq 2$ where $I_{R}$ is an identity endomorphism of $R$. We also know that there exists a $2 \times 2$ full (and also upper triangular) matrix ring $R$ with an endomorphism $\alpha$ such that $R$ is not $\alpha$-Armendariz by [6, Theorem 1.8] and [5, Example 13] in general. Hence, we consider the following.

A ring $R$ can be extended to a ring

$$
S_{3}(R)=\left\{\left(\begin{array}{ccc}
a & b & c \\
0 & a & d \\
0 & 0 & a
\end{array}\right) \mid a, b, c, d \in R\right\}
$$

and an endomorphism $\alpha$ of $R$ can also be extended to the endomorphism $\bar{\alpha}$ : $S_{3}(R) \rightarrow S_{3}(R)$ defined by $\bar{\alpha}\left(\left(a_{i j}\right)\right)=\left(\alpha\left(a_{i j}\right)\right)$. Recall that the trivial extension $T(R, M)=R \oplus M$ of $R$ by $M$ is isomorphic to the ring of all matrices $\left(\begin{array}{ll}r & m \\ 0 & r\end{array}\right)$, where $r \in R$ and $m \in M$ and the usual matrix operations are used. Hong et al. [6, Proposition 2.1] proved that, if $R$ is an $\alpha$-rigid ring, then $S_{3}(R)$ is $\bar{\alpha}$-Armendariz and so the trivial extension $T(R, R)$ of $R$ is $\bar{\alpha}$-Armendariz. Now, we show that these are equivalent. First we state the following lemma.

Lemma 1. Let $\alpha$ be an endomorphism of a ring $R$.

(1) [6, Proposition 1.3(ii)] If $R$ is an $\alpha$-Armendariz ring, then $\alpha$ is a monomorphism.

(2) [6, Proposition 2.4] $R$ is an $\alpha$-rigid ring if and only if for each $a \in R$, $\alpha^{a}(a) a=0$ implies $a=0$.

Theorem 2. Let $\alpha$ be an endomorphism of a ring $R$. Then the following are equivalent:

(1) $R$ is an $\alpha$-rigid ring.

(2) $S_{3}(R)=\left\{\left(\begin{array}{lll}a & b & c \\ 0 & a & d \\ 0 & 0 & a\end{array}\right) \mid a, b, c, d \in R\right\}$ is an $\bar{\alpha}$-Armendariz ring.

(3) The trivial extension $T(R, R)$ of $R$ is an $\bar{\alpha}$-Armendariz ring. 
Proof. Note that $T(R, R)$ is isomorphic to the subring

$$
\left\{\left(\begin{array}{ccc}
a & b & 0 \\
0 & a & 0 \\
0 & 0 & a
\end{array}\right) \mid a, b \in R\right\}
$$

of a ring $S_{3}(R)$ and each subring of an $\alpha$-Armendariz ring is also $\alpha$-Armendariz. Hence, it is enough to show that $(3) \Rightarrow(1)$. Let $T(R, R)$ be $\bar{\alpha}$-Armendariz. Assume on the contrary that $R$ is not $\alpha$-rigid. By Lemma 1, there exists $0 \neq a \in R$ such that $\alpha(a) a=0$ and $\alpha(a) \neq 0$. For $p(x)=\left(\begin{array}{cc}\alpha(a) & 0 \\ 0 & \alpha(a)\end{array}\right)+$ $\left(\begin{array}{ll}0 & 1 \\ 0 & 0\end{array}\right) x, \quad q(x)=\left(\begin{array}{cc}a & 0 \\ 0 & a\end{array}\right)+\left(\begin{array}{cc}0 & -1 \\ 0 & 0\end{array}\right) x \in T(R, R)[x ; \bar{\alpha}]$, we have $p(x) q(x)=0$, but $\left(\begin{array}{cc}\alpha(a) & 0 \\ 0 & \alpha(a)\end{array}\right)\left(\begin{array}{cc}0 & -1 \\ 0 & 0\end{array}\right) \neq 0$; which is a contradiction. Thus $R$ is $\alpha$-rigid.

If we take $\alpha$ as the identity endomorphism $I_{R}$ of a $\operatorname{ring} R$, then we have the following corollary which generalizes the results in [8, Proposition 2] and [10, Theorem 2.3].

Corollary 3. For a ring $R$, the following are equivalent:

(1) $R$ is a reduced ring.

(2) $S_{3}(R)=\left\{\left(\begin{array}{lll}a & b & c \\ 0 & a & d \\ 0 & 0 & a\end{array}\right) \mid a, b, c, d \in R\right\}$ is an Armendariz ring.

(3) The trivial extension $T(R, R)$ of $R$ is an Armendariz ring.

Hong et al. [5, p. 261] showed that the ring

$$
S_{n}(R)=\left\{\left(\begin{array}{ccccc}
a & a_{12} & a_{13} & \cdots & a_{1 n} \\
0 & a & a_{23} & \cdots & a_{2 n} \\
0 & 0 & a & \cdots & a_{3 n} \\
\vdots & \vdots & \vdots & \ddots & \vdots \\
0 & 0 & 0 & \cdots & a
\end{array}\right) \mid a, a_{i j} \in R\right\}
$$

cannot be $\bar{\alpha}$-Armendariz for $n \geq 4$, even if $R$ is an $\alpha$-rigid ring. However, we obtain subrings of $S_{n}(R)$ for $n \geq 4$ which are $\bar{\alpha}$-Armendariz as follows.

From [11], $R A=\{r A \mid r \in R\}$ for any $A \in \operatorname{Mat}_{n}(R)$ where $\operatorname{Mat}_{n}(R)$ is the $n \times n$ full matrix ring and for $n \geq 2$, let $V=\sum_{i=1}^{n-1} E_{i(i+1)}$ where $E_{i j}$ 's are the matrix units. For an even number $n=2 k(\geq 2)$, let

$$
A_{n}^{e}(R)=\sum_{i=1}^{k} \sum_{j=k+i}^{n} R E_{i j}, \quad \text { and } \quad B_{n}^{e}(R)=\sum_{i=1}^{k+1} \sum_{j=k+i-1}^{n} R E_{i j} ;
$$

and for an odd number $n=2 k+1(\geq 3)$, let

$$
A_{n}^{o}(R)=\sum_{i=1}^{k+1} \sum_{j=k+i}^{n} R E_{i j}, \quad \text { and } \quad B_{n}^{o}(R)=\sum_{i=1}^{k+2} \sum_{j=k+i-1}^{n} R E_{i j} .
$$

In addition, for $n \geq 2$ put

$$
\begin{aligned}
& A_{n}(R)=R I_{n}+R V+\cdots+R V^{k-1}+A_{n}^{e}(R) \text { and } \\
& B_{n}(R)=R I_{n}+R V+\cdots+R V^{k-2}+B_{n}^{e}(R) \text { for } n=2 k ;
\end{aligned}
$$


$A_{n}(R)=R I_{n}+R V+\cdots+R V^{k-1}+A_{n}^{o}(R)$ and

$B_{n}(R)=R I_{n}+R V+\cdots+R V^{k-2}+B_{n}^{o}(R)$ for $n=2 k+1$, where $I_{n}$ is the unit matrix of $\operatorname{Mat}_{n}(R)$.

Proposition 4. Let $\alpha$ be an endomorphism of a ring $R$. The following are equivalent:

(1) $R$ is an $\alpha$-rigid ring.

(2) $A_{n}(R)$ is an $\bar{\alpha}$-Armendariz ring for $n=2 k+1 \geq 3$.

(3) $A_{n}(R)+R E_{1 k}$ is an $\bar{\alpha}$-Armendariz ring for $n=2 k \geq 4$.

(4) $V_{n}(R)=R I_{n}+R V+R V^{2}+\cdots+R V^{n-1}$ is an $\bar{\alpha}$-Armendariz ring for $n \geq 2$.

Proof. Assume that $R$ is an $\alpha$-rigid ring. First, let $S=A_{n}(R)$ if $n=2 k+1 \geq 3$ and $S=A_{n}(R)+R E_{1 k}$ if $n=2 k \geq 4$, and let $P(x)=C_{0}+C_{1} x+\cdots+C_{u} x^{u}$ and $Q(x)=D_{0}+D_{1} x+\cdots+D_{v} x^{v}$ in $S[x ; \bar{\alpha}]$ with $P(x) Q(x)=0$. We show that $C_{i} D_{j}=0$ for $0 \leq i \leq u, 0 \leq j \leq v$. Let $p_{s t}(x)=c_{s t}^{(0)}+c_{s t}^{(1)} x+\cdots+c_{s t}^{(u)} x^{u}$ and $q_{s t}(x)=d_{s t}^{(0)}+d_{s t}^{(1)} x+\cdots+d_{s t}^{(v)} x^{v}$, where $c_{s t}^{(i)}$ and $d_{s t}^{(j)}$ are the $(s, t)$ entries of $C_{i}$ and $D_{j}$, respectively for $0 \leq i \leq u$ and $0 \leq j \leq v$. Then, we can write that $P(x)=\left(p_{s t}(x)\right)$ and $Q(x)=\left(q_{s t}(x)\right)$ for $1 \leq s, t \leq n$ and then $\left(p_{s t}(x)\right)\left(q_{s t}(x)\right)=0$ in $S$. Since $R$ is $\alpha$-rigid, $R[x ; \alpha]$ is reduced by [5, Proposition 3]. From [3, Lemma 2.4], we have $\left(\left(p_{s t}(x)\right)\left(q_{s t}(x)\right)\right)_{s t}=0$ for $1 \leq s, t \leq n$. So $p_{s l}(x) q_{l t}(x)=0$ in $R[x ; \alpha]$ for $1 \leq l \leq n$. That is,

$$
\left(c_{s l}^{(0)}+c_{s l}^{(1)} x+\cdots+c_{s l}^{(u)} x^{u}\right)\left(d_{l t}^{(0)}+d_{l t}^{(1)} x+\cdots+d_{l t}^{(v)} x^{v}\right)=0
$$

Since $R$ is an $\alpha$-rigid, $R$ is $\alpha$-Armendariz by [6, Proposition 1.7]. Hence $c_{s l}^{(i)} d_{l t}^{(j)}=0$ for $1 \leq s, t, l \leq n, 0 \leq i \leq u$ and $0 \leq j \leq v$, and so $C_{i} D_{j}=$ $\left(c_{s t}^{(i)}\right)\left(d_{s t}^{(j)}\right)=0$. Therefore $S$ is $\bar{\alpha}$-Armendariz. This proves that $(1) \Rightarrow(2)$ and (1) $\Rightarrow(3)$.

Next, assume that $R$ is an $\alpha$-rigid ring. For $n=2,3, V_{n}(R)$ is $\bar{\alpha}$-Armendariz by Theorem 2 and for $n \geq 4, V_{n}(R)$ is also $\bar{\alpha}$-Armendariz since $V_{n}(R)$ is a subring of $A_{n}(R)$ or $A_{n}(R)+R E_{1 k}$ and $\alpha\left(V_{n}(R)\right) \subseteq V_{n}(R)$.

The converses follow the proof of Theorem 2, respectively.

Corollary 5. The following are equivalent for a ring $R$.

(1) $R$ is a reduced ring.

(2) $A_{n}(R)$ is an Armendariz ring for $n=2 k+1 \geq 3$.

(3) $A_{n}(R)+R E_{1 k}$ is an Armendariz ring for $n=2 k \geq 4$.

(4) $V_{n}(R)=R I_{n}+R V+R V^{2}+\cdots+R V^{n-1}$ is an Armendariz ring for $n \geq 2$.

If we define $\rho: V_{n}(R) \rightarrow R[x] /\left\langle x^{n}\right\rangle$ by $\rho\left(a_{0} I_{n}+a_{1} V+\cdots+a_{n-1} V^{n-1}\right)=$ $a_{0}+a_{1} x+\cdots+a_{n-1} x^{n-1}+\left\langle x^{n}\right\rangle$, then $\rho$ is a ring isomorphism, where $\left\langle x^{n}\right\rangle$ is an ideal of $R[x]$ generated by $x^{n}$ and $n \geq 2$. So we have the following corollary. 
Corollary 6 ([6, Proposition 2.4]). Let $\alpha$ be an endomorphism of a ring $R$. Then the factor ring $R[x] /\left\langle x^{n}\right\rangle$ is $\bar{\alpha}$-Armendariz if and only if $R$ is an $\alpha$-rigid ring.

Remark 7 . Observe that $B_{n}^{e}(R)$ for $n=2 k \geq 2, B_{n}^{o}(R)$ for $n=2 k+1 \geq 3$ and $B_{n}(R)$ for $n \geq 2$ are not $\bar{\alpha}$-Armendariz rings, even though $R$ is an $\alpha$-Armendariz ring by the same method as in [11, Example 1.1], since the endomorphism $\alpha$ of an $\alpha$-Armendariz ring $R$ preserves identity by [6, Corollary 1.4(i)].

The following example shows that there exists an Armendariz ring $R$ with an endomorphism $\alpha$ such that $R$ is not $\alpha$-skew Armendariz.

Example 8. Let $R=\mathbb{Z}_{2} \oplus \mathbb{Z}_{2}$ where $\mathbb{Z}_{2}$ is the ring of integers modulo 2. Then $R$ is a commutative reduced ring, and so it is Armendariz. Let $\alpha: R \rightarrow R$ be an endomorphism defined by $\alpha((a, b))=(b, a)$. Then $R$ is not $\alpha$-skew Armendariz by [5, Example 2], and so $R$ is not $\alpha$-Armendariz, either by [6, Theorem 1.8].

However, we have the following.

Proposition 9. Let $\alpha$ be an endomorphism of a ring $R$. If the skew polynomial ring $R[x ; \alpha]$ of $R$ is an Armendariz ring, then $R$ is $\alpha$-skew Armendariz.

Proof. Assume that $R[x ; \alpha]$ is Armendariz. Let $p(x) q(x)=0$, where $p(x)=$ $a_{0}+a_{1} x+\cdots+a_{m} x^{m}$ and $q(x)=b_{0}+b_{1} x+\cdots+b_{n} x^{n}$ in $R[x ; \alpha]$. We show that $a_{i} \alpha^{i}\left(b_{j}\right)=0$ for all $i, j$. Set $f(y)=a_{0}+\left(a_{1} x\right) y+\cdots+\left(a_{m} x^{m}\right) y^{m}$ and $g(y)=b_{0}+\left(b_{1} x\right) y+\cdots+\left(b_{n} x^{n}\right) y^{n}$ in $(R[x ; \alpha])[y]$. Then $f(y) g(y)=0$, since $p(x) q(x)=0$ and $y$ commutes with $x$. Since $R[x ; \alpha]$ is Armendariz, we have $a_{i} x^{i} b_{j} x^{j}=0$, and so $a_{i} \alpha^{i}\left(b_{j}\right)=0$ for all $i, j$. Therefore $R$ is an $\alpha$-skew Armendariz ring.

Corollary 10 ([5, Corollary 4]). Let $\alpha$ be an endomorphism of a ring $R$. If $R$ is $\alpha$-rigid, then $R$ is an $\alpha$-skew Armendariz ring.

Proof. Let $R$ be an $\alpha$-rigid ring. Then $R[x ; \alpha]$ is reduced by [5, Proposition 3] and so $R[x ; \alpha]$ is Armendariz. Therefore $R$ is an $\alpha$-skew Armendariz ring by Proposition 9 .

Observe that the conclusion of Proposition 9 cannot be replaced by the condition " $R$ is $\alpha$-Armendariz" by the next example.

Example 11. Let $R$ be the polynomial ring $\mathbb{Z}_{2}[x]$ over $\mathbb{Z}_{2}$, the ring of integers modulo 2, and let the endomorphism $\alpha$ of $R$ be defined by $\alpha(f(x))=f(0)$ for $f(x) \in \mathbb{Z}_{2}[x]$. Then $R$ is a reduced $\alpha$-skew Armendariz ring by [5, Example 5], but $R$ is not $\alpha$-Armendariz by [6, Example 1.9]. Now, we show that $S=R[y ; \alpha]$ is an Armendariz ring. Let $f(T)=f_{0}+f_{1} T+\cdots+f_{m} T^{m}$ and $g(T)=g_{0}+$ $g_{1} T+\cdots+g_{n} T^{n} \in S[T]$ with $f(T) g(T)=0$. We also let $f_{i}=\sum_{s=0}^{u_{i}} f_{i_{s}}(x) y^{s}$ and $g_{j}=\sum_{t=0}^{v_{j}} g_{j_{t}}(x) y^{t}$ where $f_{i_{s}}(x), g_{j_{t}}(x) \in \mathbb{Z}_{2}[x]$ for each $0 \leq i \leq m$ and $0 \leq j \leq n$. Without loss of generality, assume that $f_{i_{s}}(x) \neq 0$ and $g_{j_{t}}(x) \neq 0$ 
in $\mathbb{Z}_{2}[x]$ for all $1 \leq s \leq u_{i}, 0 \leq t \leq v_{j}, 0 \leq i \leq m$ and $0 \leq j \leq n$. Then we have the following system of equations:

(0) $f_{0} g_{0}=0$;

(1) $f_{0} g_{1}+f_{1} g_{0}=0$;

(k) $f_{0} g_{k}+f_{1} g_{k-1}+\cdots+f_{k-1} g_{1}+f_{k} g_{0}=0$;

$(k+1) f_{0} g_{k+1}+f_{1} g_{k}+\cdots+f_{k} g_{1}+f_{k+1} g_{0}=0 ;$

$$
(m+n) f_{m} g_{n}=0 \text {. }
$$

We claim that $f_{0_{0}}(x)=f_{1_{0}}(x)=\cdots=f_{m_{0}}(x)=0$ and each $g_{j_{t}}(x)$ has no constant term for $0 \leq t \leq v_{j}$ and $0 \leq j \leq n$. We proceed by induction on $i+j$. Since $R$ is $\alpha$-skew Armendariz and $f_{0} g_{0}=0$ from Eq.(0), we obtain $f_{0_{s}}(x) \alpha^{s}\left(g_{0_{t}}(x)\right)=0$ for all $0 \leq s \leq u_{0}$ and $0 \leq t \leq v_{0}$, and so $f_{0_{0}}(x)=0$ and $g_{0_{t}}(0)=0$ for $0 \leq t \leq v_{0}$. So each $g_{0_{t}}(x)$ has no constant term for $0 \leq t \leq v_{0}$. This proves for $i+j=0$. Now suppose that our claim is true for $i+j \leq k-1$. By the induction hypothesis and Eq. $(k)$, we get $0=f_{0} g_{k}+f_{k} g_{0}=$ $\left(f_{0_{1}}(x) y+f_{0_{2}}(x) y^{2}+\cdots+f_{0_{u_{0}}}(x) y^{u_{0}}\right)\left(g_{k_{0}}(x)+g_{k_{1}}(x) y+\cdots+g_{k_{v_{k}}}(x) y^{v_{k}}\right)+$ $f_{k_{0}}(x)\left(g_{0_{0}}(x)+g_{0_{1}}(x) y+\cdots+g_{0_{v_{0}}}(x) y^{v_{0}}\right)=f_{k_{0}}(x) g_{0_{0}}(x)+\left[f_{k_{0}}(x) g_{0_{1}}(x)+\right.$ $\left.f_{0_{1}}(x) \alpha\left(g_{k_{0}}(x)\right)\right] y+\left[f_{k_{0}}(x) g_{0_{2}}(x)+f_{0_{1}}(x) \alpha\left(g_{k_{1}}(x)\right)+f_{0_{2}}(x) \alpha^{2}\left(g_{k_{0}}(x)\right)\right] y^{2}+\cdots+$ $f_{0_{u_{0}}}(x) \alpha^{u_{0}}\left(g_{k_{v_{k}}}(x)\right) y^{u_{0}+v_{k}}$. Then $f_{k_{0}}(x)=0$, and so we have the following:

(i) $f_{0_{1}}(x) \alpha\left(g_{k_{0}}(x)\right)=0$;

(ii) $f_{0_{1}}(x) \alpha\left(g_{k_{1}}(x)\right)+f_{0_{2}}(x) \alpha^{2}\left(g_{k_{0}}(x)\right)=0$;

(iii) $f_{0_{1}}(x) \alpha\left(g_{k_{i-1}}(x)\right)+f_{0_{2}}(x) \alpha^{2}\left(g_{k_{i-2}}(x)\right)+\cdots+f_{0_{i}}(x) \alpha^{i}\left(g_{k_{0}}(x)\right)=0$;

(iv) $f_{0_{u_{0}}}(x) \alpha^{u_{0}}\left(g_{k_{v_{k}}}(x)\right)=0$.

Hence, $g_{k_{0}}(0)=g_{k_{1}}(0)=\cdots=g_{k_{v_{k}}}(0)=0$, and so $g_{k_{t}}(x)$ has no constant term for all $0 \leq t \leq v_{k}$. Thus $f_{i}=\sum_{s=1}^{u_{i}} f_{i_{s}}(x) y^{s}, g_{j}=\sum_{t=0}^{v_{j}} g_{j_{t}}(x) y^{t}$ and each $g_{j_{t}}$ has no constant term for $0 \leq i \leq m, 0 \leq t \leq v_{j}$ and $0 \leq j \leq n$, and so $f_{i} g_{j}=0$ for all $i, j$. Therefore $S=R[y ; \alpha]=\left(\mathbb{Z}_{2}[x]\right)[y ; \alpha]$ is Armendariz.

The following extends the result in [3, Lemma 3.8].

Proposition 12. Let $\alpha$ be an endomorphism of a ring $R$. If $S$ is a ring and $\sigma: R \rightarrow S$ is a ring isomorphism, then we have the following.

(1) $R$ is an $\alpha$-rigid ring if and only if $S$ is a $\sigma \alpha \sigma^{-1}$-rigid ring.

(2) $R$ is an $\alpha$-Armendariz ring if and only if $S$ is a $\sigma \alpha \sigma^{-1}$-Armendariz ring.

(3) $R$ is an $\alpha$-skew Armendariz ring if and only if $S$ is a $\sigma \alpha \sigma^{-1}$-skew Armendariz ring. 
Proof. (1) For $a \in R$, there exists $a^{\prime} \in S$ such that $\sigma(a)=a^{\prime}$ since $\sigma$ is bijective, and so $a \alpha(a)=0$ if and only if $\sigma(a)\left(\sigma \alpha \sigma^{-1}\right) \sigma(a)=0$ if and only if $a^{\prime}\left(\sigma \alpha \sigma^{-1}\right)\left(a^{\prime}\right)=0$. This yields that $R$ is $\alpha$-rigid if and only if $S$ is $\sigma \alpha \sigma^{-1}$-rigid.

(2) and (3) Similarly, $p(x)=\sum_{i=0}^{m} a_{i} x^{i}, q(x)=\sum_{j=0}^{n} b_{j} x^{j} \in R[x ; \alpha]$ if and only if $p^{\prime}(x)=\sum_{i=0}^{m} a_{i}^{\prime} x^{i}, q^{\prime}(x)=\sum_{j=0}^{n} b_{j}^{\prime} x^{j} \in S\left[x ; \sigma \alpha \sigma^{-1}\right]$, letting $\sigma\left(a_{i}\right)=a_{i}^{\prime}, \sigma\left(b_{j}\right)=b_{j}^{\prime}$ for all $i, j$ since $\sigma$ is bijective. Then $p(x) q(x)=0$ in $R[x ; \alpha]$ if and only if $\sum_{i+j=k} a_{i} \alpha^{i}\left(b_{j}\right)=0$ for each $0 \leq k \leq m+n$ if and only if $\sum_{i+j=k} \sigma\left(a_{i} \alpha^{i}\left(b_{j}\right)\right)=0$ for each $0 \leq k \leq m+n$ if and only if $\sum_{i+j=k} \sigma\left(a_{i}\right)\left(\sigma \alpha \sigma^{-1}\right)^{i} \sigma\left(b_{j}\right)=0$ for each $0 \leq k \leq m+n$, since $\left(\sigma \alpha \sigma^{-1}\right)^{t}=$ $\sigma \alpha^{t} \sigma^{-1}$ for any positive integer $t$ if and only if $\sum_{i+j=k} a_{i}^{\prime}\left(\sigma \alpha \sigma^{-1}\right)^{i}\left(b_{j}^{\prime}\right)=0$ for each $0 \leq k \leq m+n$ if and only if $p^{\prime}(x) q^{\prime}(x)=0$ in $S\left[x ; \sigma \alpha \sigma^{-1}\right]$. Hence, for all $i, j, a_{i} b_{j}=0$ if and only if $a_{i}^{\prime} b_{j}^{\prime}=0$; and $a_{i} \alpha^{i}\left(b_{j}\right)=0$ if and only if $\sigma\left(a_{i}\right)\left(\sigma \alpha \sigma^{-1}\right)^{i} \sigma\left(b_{j}\right)=0$ if and only if $a_{i}^{\prime}\left(\sigma \alpha \sigma^{-1}\right)^{i}\left(b_{j}^{\prime}\right)=0$. The proof is completed.

Recall that if $\alpha$ is an endomorphism of a ring $R$, then the map $\bar{\alpha}: R[x] \rightarrow$ $R[x]$ defined by $\bar{\alpha}\left(\sum_{i=0}^{m} a_{i} x^{i}\right)=\sum_{i=0}^{m} \alpha\left(a_{i}\right) x^{i}$ is an endomorphism of the polynomial ring $R[x]$ and clearly this map extends $\alpha$. The Laurent polynomial ring $R\left[x, x^{-1}\right]$ with an indeterminate $x$, consists of all formal sums $\sum_{i=k}^{n} a_{i} x^{i}$, where $a_{i} \in R$ and $k, n$ are (possibly negative) integers. The map $\bar{\alpha}: R\left[x, x^{-1}\right] \rightarrow$ $R\left[x, x^{-1}\right]$ defined by $\bar{\alpha}\left(\sum_{i=k}^{n} a_{i} x^{i}\right)=\sum_{i=k}^{n} \alpha\left(a_{i}\right) x^{i}$ extends $\alpha$ and also is an endomorphism of $R\left[x, x^{-1}\right]$.

Theorem 13. Let $\alpha$ be an endomorphism of a ring $R$. The following are equivalent:

(1) $R$ is an $\alpha$-rigid ring.

(2) $R[x]$ is an $\bar{\alpha}$-rigid ring.

(3) $R\left[x, x^{-1}\right]$ is an $\bar{\alpha}$-rigid ring.

Proof. $(1) \Rightarrow(2)$ Assume that $R$ is $\alpha$-rigid, but $R[x]$ is not $\bar{\alpha}$-rigid. Then there exists a nonzero $f(x)=\sum_{i=0}^{n} a_{i} x^{i} \in R[x]$ such that $f(x) \bar{\alpha}(f(x))=0$. Suppose that $a_{k} \neq 0$ and $a_{0}=\cdots=a_{k-1}=0$ where $0 \leq k \leq n$. Then $0=f(x) \bar{\alpha}(f(x))=\left(a_{k} x^{k}+\cdots+a_{n} x^{n}\right)\left(\alpha\left(a_{k}\right) x^{k}+\cdots+\alpha\left(a_{n}\right) x^{n}\right)$ yields $a_{k} \alpha\left(a_{k}\right)=0$, and so $a_{k}=0$; which is a contradiction. Thus $R[x]$ is $\bar{\alpha}$-rigid.

$(2) \Rightarrow(3)$ Let $f(x) \in R\left[x, x^{-1}\right]$ with $f(x) \bar{\alpha}(f(x))=0$. Then there exists a positive integer $n$ such that $f_{1}(x)=f(x) x^{n} \in R[x]$, and so $f_{1}(x) \bar{\alpha}\left(f_{1}(x)\right)=0$. Since $R[x]$ is $\bar{\alpha}$-rigid, we obtain $f_{1}(x)=0$, and hence $f(x)=0$. Thus $R\left[x, x^{-1}\right]$ is $\bar{\alpha}$-rigid.

$(3) \Rightarrow(1) R$ is $\alpha$-rigid as a subring of $R\left[x, x^{-1}\right]$ when $R$ is $\bar{\alpha}$-rigid.

Corollary 14. (1) Let $R$ be a reduced ring with an endomorphism $\alpha$. Then $R$ is $\alpha$-Armendariz if and only if $R[x]$ is $\bar{\alpha}$-Armendariz.

(2) [2, Proposition 6] Let $R$ be a reduced ring and $\alpha$ be a monomorphism of $R$. Then $R$ is $\alpha$-skew Armendariz if and only if $R[x]$ is $\bar{\alpha}$-skew Armendariz. 
Proof. It follows from [2, Theorem 1], [6, Proposition 1.7] and Theorem 13.

Related to Corollary 14, notice that there exists a reduced $\alpha$-skew Armendariz ring which is not $\alpha$-Armendariz (Example 11).

Let $\alpha_{\gamma}$ be an endomorphism of a ring $R_{\gamma}$ for each $\gamma \in \Gamma$. For the product $\prod_{\gamma \in \Gamma} R_{\gamma}$ of $R_{\gamma}$ and the endomorphism $\bar{\alpha}: \prod_{\gamma \in \Gamma} R_{\gamma} \rightarrow \prod_{\gamma \in \Gamma} R_{\gamma}$ defined by $\bar{\alpha}\left(\left(a_{\gamma}\right)\right)=\left(\alpha_{\gamma}\left(a_{\gamma}\right)\right)$, it can be easily checked that $\prod_{\gamma \in \Gamma} R_{\gamma}$ is $\bar{\alpha}$-rigid if and only if each $R_{\gamma}$ is $\alpha_{\gamma}$-rigid.

Recall that for an endomorphism $\alpha$ and an ideal $I$ of a ring $R, I$ is called an $\alpha$-ideal if $\alpha(I) \subseteq I$, and if $I$ is an $\alpha$-ideal of $R$, then $\bar{\alpha}: R / I \rightarrow R / I$ defined by $\bar{\alpha}(a+I)=\alpha(a)+I$ for $a \in R$ is an endomorphism of the factor ring $R / I$. The homomorphic image of an $\alpha$-rigid ring is not $\bar{\alpha}$-rigid, in general. The following example shows that there exists a ring $R$ with an automorphism $\alpha$ such that $R / I$ is $\bar{\alpha}$-rigid for a non-zero $\alpha$-ideal $I$ of $R$, but $R$ is not $\alpha$-rigid.

Example 15. Let $R=\left(\begin{array}{cc}F & F \\ 0 & F\end{array}\right)$ where $F$ is a field, and $\alpha$ be defined by $\alpha\left(\left(\begin{array}{ll}a & b \\ 0 & c\end{array}\right)\right)$ $=\left(\begin{array}{cc}a & -b \\ 0 & c\end{array}\right)$. Note that $R$ is not $\alpha$-Armendariz by [6, Example 1.12], and so it is not $\alpha$-rigid. However, for a nonzero proper ideal $I=\left(\begin{array}{ll}0 & F \\ 0 & 0\end{array}\right)$ of $R$, it can be easily checked that $\alpha(I) \subseteq I$ and $R / I$ is $\bar{\alpha}$-rigid.

Let $\alpha$ be an automorphism of a ring $R$. Suppose that there exists the classical right quotient ring $Q(R)$ of $R$. Then for any $a b^{-1} \in Q(R)$ where $a, b \in R$ with $b$ regular, the induced map $\bar{\alpha}: Q(R) \rightarrow Q(R)$ defined by $\bar{\alpha}\left(a b^{-1}\right)=\alpha(a) \alpha(b)^{-1}$ is also an endomorphism. Note that $R$ is $\alpha$-rigid if and only if $Q(R)$ is $\bar{\alpha}$-rigid.

Let $R$ be an algebra over a commutative ring $S$. Recall that the Dorroh extension of $R$ by $S$ is the ring $D=R \times S$ with operations $\left(r_{1}, s_{1}\right)+\left(r_{2}, s_{2}\right)=$ $\left(r_{1}+r_{2}, s_{1}+s_{2}\right)$ and $\left(r_{1}, s_{1}\right)\left(r_{2}, s_{2}\right)=\left(r_{1} r_{2}+s_{1} r_{2}+s_{2} r_{1}, s_{1} s_{2}\right)$, where $r_{1}, r_{2} \in R$ and $s_{1}, s_{2} \in S$. For an endomorphism $\alpha$ of $R$ and the Dorroh extension $D$ of $R$ by $S, \bar{\alpha}: D \rightarrow D$ defined by $\bar{\alpha}(r, s)=(\alpha(r), s)$ is an $S$-algebra homomorphism.

In the following, we give some other example of $\alpha$-rigid rings. Observe that for an $\alpha$-rigid ring $R$, every subring $S$ of $R$ with $\alpha(S) \subseteq S$ is clearly $\alpha$-rigid, and $R$ is reduced with $\alpha(e)=e$ for $e^{2}=e \in R$ by [4, Proposition 5].

Proposition 16. Let $\alpha$ be an endomorphism of a ring $R$.

(1) $R$ is an $\alpha$-rigid ring if and only if $e R$ and $(1-e) R$ are $\alpha$-rigid for $e^{2}=e \in R$.

(2) If $R$ is an $\alpha$-rigid ring and $S$ is a reduced ring, then the Dorroh extension $D$ of $R$ by $S$ is $\bar{\alpha}$-rigid.

Proof. (1) It is enough to show that $R$ is $\alpha$-rigid. Suppose that $e R$ and $(1-e) R$ are $\alpha$-rigid. Let $a \alpha(a)=0$ for $a \in R$. Then $0=e a \alpha(e a)$ and $0=(1-e) a \alpha((1-$ $e) a)$. By hypothesis, we get $e a=0$ and $(1-e) a=0$, and so $a=0$. Thus $R$ is $\alpha$-rigid.

(2) Let $(r, s) \in D$ with $(r, s) \bar{\alpha}(r, s)=0$. Then $r \alpha(r)+s \alpha(r)+s r=0$ and $s^{2}=0$. Since $S$ is reduced, we get $s=0$. Thus $r \alpha(r)=0$, and so $r=0$ since $R$ is $\alpha$-rigid. Hence, $(r, s)=0$, and therefore the Dorroh extension $D$ is $\bar{\alpha}$-reduced. 
For any endomorphism $\alpha$ of a ring $R, R$ is $\alpha$-rigid if and only if $R[x ; \alpha]$ is reduced by [5, Proposition 3], but there exists a semiprime ring $R$ with an automorphism $\alpha$ such that the skew polynomial ring $R[x ; \alpha]$ is not semiprime by the following example.

Example 17. Let $F$ be a field and $F_{i}=F$ for $i \in \mathbb{Z}$. Let $R$ be a $F$-subalgebra of $\prod_{i \in \mathbb{Z}} F_{i}$ generated by $\oplus_{i \in \mathbb{Z}} F_{i}$ and $1_{\prod_{i \in \mathbb{Z}} F_{i}}$. Let $\alpha$ be an automorphism of $R$ defined by $\alpha\left(\left(a_{i}\right)\right)=\left(a_{i+1}\right)$. Then

$$
R=\left\{\left(a_{i}\right) \in \prod_{i \in \mathbb{Z}} F_{i} \mid a_{i} \text { is eventually constant }\right\}
$$

is reduced and von Neumann regular, but $R[x ; \alpha]$ is not semiprime by $[7$, Example 4.3].

Lemma 18. For a ring $R$, the following are equivalent:

(1) $R$ is a semiprime ring.

(2) For $a, b \in R, a R b=0$ implies $a R \cap R b=0$.

Proof. Suppose that $R$ is semiprime and $a R b=0$ for $a, b \in R$. Let $c \in a R \cap R b$. Then $c=a r=s b$ for some $r, s \in R$. So $c R c=(a r) R(s b) \subseteq a R b=0$, and thus $c=0$. The converse is obvious.

A ring $R$ is called semicommutative if $a b=0$ implies $a R b=0$ for $a, b \in R$; and so every reduced ring is semicommutative.

Theorem 19. Let $\alpha$ be an endomorphism of a ring $R$. The following are equivalent:

(1) $R$ is an $\alpha$-rigid ring. 0 .

(2) For $p(x), q(x) \in R[x ; \alpha], p(x) q(x)=0$ implies $p(x) R[x ; \alpha] \cap R[x ; \alpha] q(x)=$

Proof. Assume that $R$ is $\alpha$-rigid. By [5, Proposition 3], $R[x ; \alpha]$ is reduced and so it is semiprime and semicommutative. If $p(x) q(x)=0$ for $p(x), q(x) \in R[x ; \alpha]$, then $p(x) R[x ; \alpha] q(x)=0$, and thus $p(x) R[x ; \alpha] \cap R[x ; \alpha] q(x)=0$ by Lemma 18. Conversely, assume (2). Let $a \alpha(a)=0$ for $a \in R$. For $p(x)=a x=$ $q(x) \in R[x ; \alpha], p(x) q(x)=a \alpha(a) x^{2}=0$ and so $(a x) R[x ; \alpha] \cap R[x ; \alpha](a x)=0$ by hypothesis. Then $a x=0$, and hence $a=0$. Therefore $R$ is $\alpha$-rigid.

Corollary 20. For a ring $R$, the following are equivalent:

(1) $R$ is a reduced ring.

(2) $R[x]$ is a reduced ring.

(3) For $a, b \in R, a b=0$ implies $a R \cap R b=0$.

(4) For $f(x), g(x) \in R[x], f(x) g(x)=0$ implies $f(x) R[x] \cap R[x] g(x)=0$.

Proof. It follows from Theorem 13 and Theorem 19.

Paralleled to Theorem 2 and Proposition 4 in this paper, Chen and Tong [3] proved the relationship between $\alpha$-rigid rings and $\bar{\alpha}$-skew Armendariz rings. However, we note that Theorem 19 shows that the results in [3, Theorems 3.11 
and 3.12(15)] is meaningless: In [3, Theorem 3.11], Chen and Tong claimed that the trivial extension $T(R, R)$ of a ring $R$ is $\bar{\alpha}$-skew Armendariz (equivalently, $R$ is an $\alpha$-rigid ring by [3, Theorem 3.4]) if and only if $R$ is an $\alpha$-skew Armendariz ring and for $p(x), q(x) \in R[x ; \alpha], p(x) q(x)=0$ implies $p(x) R[x ; \alpha] \cap R[x ; \alpha] q(x)=0$.

Acknowledgments. The third named author was supported by Basic Science Research Program through the National Research Foundation of Korea(NRF) funded by the Ministry of Education, Science and Technology(No. 2010-0022160).

\section{References}

[1] E. P. Armendariz, A note on extensions of Baer and P.P.-rings, J. Austral. Math. Soc. 18 (1974), 470-473.

[2] W. Chen and W. Tong, A note on skew Armendariz rings, Comm. Algebra 33 (2005), no. 4, 1137-1140.

[3] — On skew Armendariz rings and rigid rings, Houston J. Math. 33 (2007), no. 2, 341-353.

[4] C. Y. Hong, N. K. Kim, and T. K. Kwak, Ore extensions of Baer and p.p.-rings, J. Pure Appl. Algebra 151 (2000), no. 3, 215-226.

[5] - On skew Armendariz rings, Comm. Algebra 31 (2003), no. 1, 103-122.

[6] C. Y. Hong, T. K. Kwak, and S. T. Rizvi, Extensions of generalized Armendariz rings, Algebra Colloq. 13 (2006), no. 2, 253-266.

[7] A. A. M. Kamal, Some remarks on Ore extension rings, Comm. Algebra 22 (1994), no. 10, 3637-3667.

[8] N. K. Kim and Y. Lee, Armendariz rings and reduced rings, J. Algebra 223 (2000), no. $2,477-488$.

[9] J. Krempa, Some examples of reduced rings, Algebra Colloq. 3 (1996), no. 4, 289-300.

[10] T. K. Lee and T. L. Wong, On Armendariz rings, Houston J. Math. 29 (2003), no. 3, 583-593.

[11] T. K. Lee and Y. Q. Zhou, Armendariz and reduced rings, Comm. Algebra 32 (2004), no. 6, 2287-2299.

[12] M. B. Rege and S. Chhawchharia, Armendariz rings, Proc. Japan Acad. Ser. A Math. Sci. 73 (1997), no. 1, 14-17.

MuhitTin BAŞER

Department of Mathematics

KOCATEPE UNIVERSITY

Afyonkarahisar 03200, Turkey

E-mail address: mbaser@aku.edu.tr

FATMA KaYNARCA

Department of Mathematics

Kocatepe University

AFYONKARAHISAR 03200, TURKEY

E-mail address: ferol@aku.edu.tr 
TAI KeUn KWAK

Department of Mathematics

DAEJIN UNIVERSITY

POCHEON 487-711, KorEA

E-mail address: tkkwak@daejin.ac.kr 